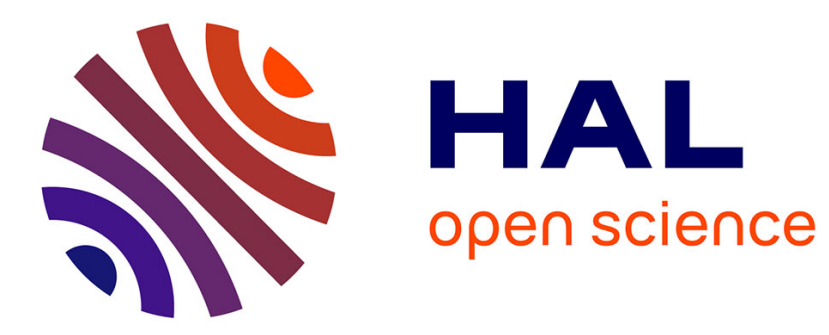

\title{
L'épigraphie médiévale
}

Robert Favreau

\section{To cite this version:}

Robert Favreau. L'épigraphie médiévale. Cahiers de Civilisation Médiévale, 1969, 12 (48), pp.393-398. 10.3406/ccmed.1969.1502 . halshs-03201774

\section{HAL Id: halshs-03201774 https://shs.hal.science/halshs-03201774}

Submitted on 19 Apr 2021

HAL is a multi-disciplinary open access archive for the deposit and dissemination of scientific research documents, whether they are published or not. The documents may come from teaching and research institutions in France or abroad, or from public or private research centers.
L'archive ouverte pluridisciplinaire HAL, est destinée au dépôt et à la diffusion de documents scientifiques de niveau recherche, publiés ou non, émanant des établissements d'enseignement et de recherche français ou étrangers, des laboratoires publics ou privés. 


\section{L'épigraphie médiévale}

Robert Favreau

\section{Citer ce document / Cite this document :}

Favreau Robert. L'épigraphie médiévale. In: Cahiers de civilisation médiévale, 12e année (n48), Octobre-décembre 1969. pp. 393-398;

doi : https://doi.org/10.3406/ccmed.1969.1502

https://www.persee.fr/doc/ccmed_0007-9731_1969_num_12_48_1502

Fichier pdf généré le 24/03/2019 


\section{MELANGES}

\section{L'épigraphie médiévale}

L'épigraphie médiévale aurait fort bien pu se constituer en science particulière dès le milieu du siècle dernier. Après la période des projets en matière d'épigraphie (au Danemark, Kellermann, en 1836; en France, Le Bas, en I835, Mérimée, en I839, le ministre Villemain, en I843 ; en Allemagne, Jahn, en I846, Mommsen, en 1847) étaient venues les grandes réalisations, Corpus inscriptionum latinarum, de Mommsen (t. Ier en I862), pour l'antiquité romaine, travaux d'Edmond Le Blant, en France, et de De Rossi, en Italie, pour les inscriptions chrétiennes antérieures au viII $\mathbf{s}$. Il n'y aurait rien eu d'étonnant si l'épigraphie médiévale avait suivi ces exemples, portée par le courant de la redécouverte romantique du moyen âge. De fait, de I834 à I84I, le marquis de Castellane publiait une vaste enquête sur les inscriptions des $v^{e}-x_{V} I^{e} s$., principalement du Midi de la France ${ }^{1}$. Le grand animateur des études archéologiques, Arcisse de Caumont, écrivait en I84I dans son Cours d'antiquités monumentales : "Les inscriptions tumulaires des $\mathrm{XI}^{\mathrm{e}}$ et $\mathrm{XII}^{\mathrm{e}} \mathrm{s}$. sont un curieux sujet d'étude que je ne saurais trop vous recommander : on a presque complètement négligé de relever ces inscriptions, dont un grand nombre a malheureusement péri ; il est grand temps de recueillir celles qui nous restent". "Dans le premier numéro des "Annales archéologiques " de Didron, en I844, Auguste Moutié publiait une étude sur les inscriptions du moyen âge et de la Renaissance, et en réclamait " un corps complet ", invitant chaque " antiquaire " à dresser des recensions épigraphiques pour sa localité, son arrondissement ${ }^{3}$. C'est à cette date qu'apparait le terme d" "épigraphie "4.

Les premiers recueils d'inscriptions eurent pour cadre le Limousin (I850), les Pyrénées-Orientales (I856I860), le Haut-Poitou (I863), le Maine-et-Loire (I868/69), l'Aunis et la Saintonge (I870) ${ }^{5}$, ce qui représente une part prépondérante pour le Centre-Ouest. Puis vinrent des recueils pour les départements du Pas-de-Calais et de la Mayenne, les anciens diocèses de Paris, Sens, Valence, l'archidiaconé d'Orléans, l'arrondissement de Vouziers, le canton normand d'Amfreville-la-Campagne, des travaux sur les villes, Autun, Vienne, Saint-Maixent ${ }^{6}$, des monuments, Saint-Benoit-sur-Loire, Saint-Bénigne de Dijon,

I. Marquis de Castellane, Inscriptions recucillies principalcment dens le Midi de la France, dans "Mém. Soc. archéol. du Midi de la France $n, t$. II, 1834/35; III, 1836/37; IV, I840/4I.

2. Arcisse de Caumont, Cours d'antiquilés monumentales..., t. VI, I81I, p. 374. Le terme "inscriptions " est attesté pour la première fois en 1 496 , et le seul employé jusclue vers le milieu du XIX $\mathbf{x}^{\mathrm{c}} \mathrm{s}$.

3. A. Movtrte, Epigraphie. Inscriptions du moyen áge ct de la Renaissancc, dans "Annales archéol. n, t. I, I844, p. 106-1 I3.

4. R. BLOCH, L'épigraphie latine, Paris, 1961 ("Que sais-je ? ", 534), p. 5, donne le mot " épigraphic " comme attesté pour la premic̀re fois ell 1845. Ie terme d' a épigraphic "placé en tête de l'article de MovTí́ en 1844 est en fait déjà cité dans le rapport présenté, le 3 août 1843 , par Figger à la Commission d'épigraphie latine désignée par le ministre Villemain.

5. Abbé H. Texıer, Manuel d'épigraphie, suivi du recueil des inscriptions du Limousin, dans "Mém. Soc. Antiq. Onest ", Ire s. t. XVIII, 1850, p. I-380, 26 h.t., fac-sim.; supplém. par E. Molinier dans Documents historiques... concernant principalement la Marche et le Limousin, t. I, 1883, p. 90-120; L. de BoNNEFoy, Epigraphie roussillonnaise, ou recueil des inscriptions des Pyrénées-Orientales, Perpignan, $1856 / 60,264$ pp., I 5 fac-sim. h.t. (d'abord paru dans * Bull. Soc. agric., scientif. et littér. des Pyr.-Orient. n); de LovGubsar, Epigraphic du Haut-Poitou, dans "Mém. Soc. Antiq. Ouest ", Ire s., t. XXVIII, r863, p. 43-399, fac-sim. dépl. h.t.; Mgr X. BARBIER DI: Montault, Epigraphie du Maine-et-Loire, dans "Répertoire histor. et archéol. de l'Anjou ", 1868, p. 105-353; 1869, p. I-1 39, h.t. L. Audiat, Epigraphie santone et aunisienne, Paris/Niort, 1870, vir-340 pp., ill., fac-sim.

6. Epigraphie du département du Pas-de-Calais, ouvr. publ. p. la Comm. départ. des Monum. histor., Arras, $1883-1932,8$ vol. ; abbé A. Avgot, Epigraphie de la Mayenne, Laval/Paris, I907, 2 vol. gr. $4^{\circ}$, ill.; Fr. de GuilherMY et R. de LASTEYrIe, Inscriptions de la France du Ve au XVIII e siècle... Ancien diocèse de Paris, Paris, I873/83, 5 vol. $4^{\circ}$, fac-sim., h.t. (" Docum. inéd. sur l'hist. de France ") ; P. Quesvers et Henri STEn, Inscriptions de l'ancien diocèse de Sens, publices d'aprís les estampages d'Edmond Michel, Paris, Picard, $\mathbf{x} 887 \cdot 1903,3$ vol., $\mathrm{x} \cdot 768,787$ et $792 \mathrm{pp}$.; C. Perrossine, Recueil des inscriptions du diacése de Valence, dans "Bull. d'Hist. ecclés. et d'archéol. relig. des dioc. de Valence, (jap, Grenoble et V'iviers", t. II, I881/82, p. 93-109, I4I-I5I, 2 II-222; t. VII, I887/88, p. 108-I17, 200-208 (inachevé). I. Mrchis, Inscriptions de l'ancien diocise d'Orléans; archidiaconé d'Orlíans..., Orléans, 1885, 202 pp.; doct. H. VLCENT, Inscriptions anciennes de l'arrondissement de Vouziers ou relatives à la région, Reims, $1892, \mathrm{xxxI-503} \mathrm{pp.,} \mathrm{ill.} \mathrm{h.t.,} \mathrm{fac-sim.} \mathrm{;}$ 
Notre-I)ane de Reims ${ }^{7}$, des périodes ${ }^{8}$, des catégories d'inscriptions, héraldiques pour la Nièvre, tumulaires pour le Mortainais et campanaires pour l'Avranchin, hébraiques pour l'ensemble de la France'. Il faut y ajouter un très grand nombre d'articles, de communications sur telle ou telle inscription, notamment à l'occasion de découvertes. L'historien d'aujourd'hui n'en reste pas moins assez désarmé s'il veut étudier l'épigraphie médiévale : les travaux, pour nombreux qu'ils soient, sont très dispersés, ne couvrent qu'une très modeste partie du territoire, sont inégaux dans leur réalisation, du fait de leur ancienneté parfois, plus généralement en raison de l'absence d'harmonisation entre les différentes entreprises. L'un s'est arrêté au $\mathrm{XVI}^{\mathrm{e}}, \mathrm{l}^{\prime}$ autre au XVIII ${ }^{\mathrm{e}}$ s., celui-ci a recueilli les légendes des monnaies, celui-là a rejeté toutes les inscriptions connues par des transcriptions mais perdues par la suite. Nous sommes loin du Corpus complet rêvé en $1844 \ldots$

Les difficultés ne s'arrêtent pas là. Les travaux d'épigraphie médiévale ont jusqu'ici été surtout des inventaires, inestimables, il est vrai, du fait de la disparition de tant et tant d'inscriptions depuis le cri d'alarme déjà lancé par Arcisse de Caumont. Le domaine de l'épigraphie comme ses objectifs restent encore à définir.

La définition classique, telle qu' on la trouve dans les différents manuels d'épigraphie latine, peut s'exprimer ainsi : l'épigraphie est la science de ce qui est écrit sur une matière dure. On rejettera, sans hésitation, cette définition, pour habituelle qu'elle soit, en considérant qu'il n'est pas satisfaisant de définir une science historique à partir simplement d'une notion de support matériel. Ce fut l'erreur des bénédictins du Nouveau traité de diplomatique, au milieu du $\mathrm{XVIII}^{\mathrm{e}} \mathrm{s}^{10}$, de distinguer entre écriture lapidaire, écriture métallique et écriture des manuscrits, distinction aujourd'hui universellement rejetée par les paléographes. De même, la numismatique n'est pas la science de tous les monuments monétiformes, ce qui l'a amenée à écarter de son domaine propre les jetons, les médailles, les poids ${ }^{11}$. La définition traditionnelle manque en outre de netteté ; peut-on vraiment parler de matières durables pour l'ardoise, la cire, le verre, etc. ? Il est vrai que la définition a été donnée par des historiens de l'antiquité qui n'avaient guère à s'occuper que d'inscriptions lapidaires ${ }^{12}$.

Peut-on tenter un premier essai de définition de l'épigraphie à partir des inscriptions médiévales ?

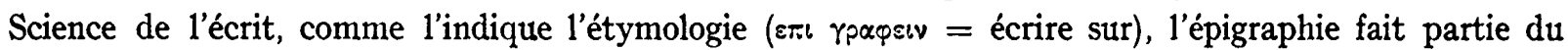
domaine large de la paléographie. Tout au plus peut-on dire que l'obligation d'écrire sur une matière habituellement plus résistante et aussi plus diversifiée peut entraîner pour la paléographie des inscriptions des caractères différant quelque peu - mais non fondamentalement - de ceux de la paléographie des chartes. L'obligation d'être lu de façon claire, parfois de loin, par beaucoup, est sans doute la cause principale de l'emploi général des majuscules dans les inscriptions.

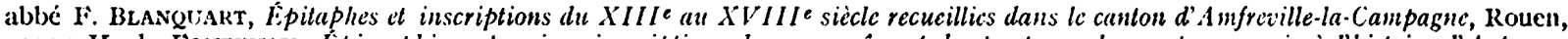
1909 ; H. de FonTENAy, Ligraphie autunoise; inscriptions du moyen age et des temps moderncs poutr scrvir a l'histoire d'A uttun..., Autum/Paris, Champion, I $883 / 86,2$ vol. $4^{\circ}$, vI- 426 et III-4I5 pp., 45 h.t. dont 2 plans dépl. (extr. de "Mím. Soc. édueune, n.s., t. VII à XIV); A. de Terrebasse, Inscriptions antiques et du moyen age de Vienne en Dauphiné. II : Inscriplions du moyen age antérieures (sic) au $X V I^{\circ}$ siècle, Vienne, I875, 2 vol. $8^{\circ}, 376$ et $472 \mathrm{pp}$., atlas $4^{\circ}$; supplément dans " Revuc du Dauphiné et du Vivarais $\mathrm{n}, \mathrm{I} 87^{8}$; I. I,EVESQUe, Inscriptions de la ville de Saint-Maixent du $X^{e}$ au $X^{\prime} I X^{e}$ siècle, dans " Mém. Soc. de Statist., sc., lettres et arts DeuxSèvres n, 1892, II 5 pp.

7. R. Boticher DE MOLAxdon, Inscriptions tumulaires des XIe et XIIe siècles à Saint-Benoit-sur-Loire..., dans "Mém. Soc. archéol. et hist. Oriéanais ", t. XVIII, r884, p. 527-573, h.t.; H. JADART, Inscriptions de Notre-Dame de Reims; textes relevés stur les monuments originaux, Reims, 1907, 280 pp., 30 fig.; G. DCMAY, Epigraphie bourguignonne; église ct abbaye de Saint-Bénigne de Dijon, Paris/Dijon, I882, $4^{\circ}, 243$ pp., h.t. (extr. de "Mém. Comm. Antiquités dép. Côte-d’Or ", t. X).

8. R. WiLL, Ripcrtoire des inscriptions romancs d'Alsace, dans " Revue d'Alsace ", t. XCVIII, I959, p. $49-84$.

9. J. de SoRnay, Epigraphie héraldique du département de la Nière, Angers, 1882, gr. $8^{\circ}$; J. Securv et li. Vivier, Corpus des inscriptions tumulaires (antc 1789) du Mortainais, et campanaires de l'Auranchin et du Mortainais, Paris, [1931/34], 96 pp., h.t., ill. ; M. Scrww B, Rapport sur les inscriptions hébraïques de la France..., Paris, $8^{\circ}, 402$ pp., fac-sim., ill. (" Nouv. archives des miss. scientif. "). La bibliographie des notes 5 à 9 est extraite des dépouillements bibliographiques réalisés par l'équipe du Corpus (cf. infra, p. 397) en $1967 / 68$.

10. Nouveau trailé de diplomatique... par deux religieux bénédictins de la congrégation de Saint-Maur, Paris, 1750/65, 6 vol. Dom TAssIN et dom Torstrars traitent de la paléographie dans les t. II et III.

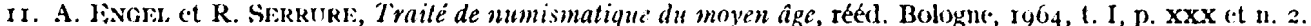

12. Mais peut-on vraiment anmexer au domaine de l'épigraphie les vingt mille tablettes d'argile du palais royal de Tell Hariri a Mari uniquement parce que ces archives royales sont ecrites sur argile? 
Les inscriptions médiévales n'ont pas pour but de fonder des droits, d'établir des actes de nature juridique, des contrats, des pièces ayant une valeur déterminée dans le temps. Elles ne constituent pas des " archives ", des sources documentaires. L'existence d'une douzaine de chartes dites "lapidaires " ne doit pas prêter à confusion, non plus que celle des rares chartes métalliques connues. I es " chartes lapidaires " sont en fait des copies, souvent abrégées - sans les préambules, les noms des témoins, - d'actes originaux faisant seuls foi en cas de contestation ${ }^{13}$. L'épigraphie se rattache plutôt à l'étude des sources narratives.

Elle a pour but de porter un élément d'information quelconque à la connaissance du public pour le présent et pour l'avenir. La notion de durée, retenue pour la définition de l'épigraphie, apparait bien plus valable dans le domaine du fond que dans celui de la forme : c'est parce qu'on souhaitait un témoignage durable qu'on a employé une matière durable. Ires notions de durée et de publicité apparaissent ici fondamentales.

On pourrait donc proposer comme nouvelle définition de l'épigraphie : science de ce qui est écrit, en général surr une matière résistante, en vue d'une publicité universelle et durable. La notion de matière pourrait être maintenue afin d'assurer une liaison avec la définition traditionnelle, mais on doit bien admettre que le support matériel de l'inscription est, en tant que tel, indifférent.

Les objectifs de l'épigraphie proprement médiévale sont, eux aussi, à préciser. Le grand archéologue Robert de Lasteyrie s'intéressa tôt à l'épigraphie. En 1877 il présenta à un concours de l'Académie des Inscriptions et Belles Lettres une étude sur les inscriptions de la France antérieures à I 108, étude malheureusement restée inédite; on ignore où se trouve le manuscrit. Il a utilisé notamment son expérience de l'épigraphie dans son travail sur le cloître de Saint-Trophime d'Arles, où il déclare : "Malheureusement l'épigraphie du moyen âge n'est guère en honneur parmi nos archéologues et je ne m'étonne pas qu'on ait si peu songé à discuter les inscriptions du cloître de Saint-Trophime. Et pourtant elles nous apportent un témoignage d'une grande valeur et permettent de déterminer avec une approximation très suffisante l'âge de ce cloître, ou du moins de la partie la plus ancienne de ses galeries ${ }^{14}$. "Sa démonstration indique, à l'évidence, que l'épigraphie était pour lui d'abord un moyen de dater les monuments. C'est aussi, me semble-t-il, la position de M. Paul Deschamps, à qui nous devons une Étude sur la paléographie des inscriptions lapidaires de la fin de l'époque mérovingienne aux dernières années $d u X I I^{e}$ siècle $^{15}$, travail qui a tant contribué à dissiper, chez les archéologues, cette ignorance de l'épigraphie que déplorait $R$. de Lasteyrie. Ce n'est pas en diminuer le mérite que de constater qu'il considère surtout l'épigraphie comme une science auxiliaire de l'archéologie, notamment pour le $\mathrm{XII}^{\mathrm{e}} \mathrm{s}$. où se fait le passage de l'art roman à l'art gothique, puisqu'il s'agit précisément d'une étude paléographique et d'inscriptions lapidaires. Plus récemment, en Belgique, M. Jacques Stiennon, faisant le point sur les études d'épigraphie médiévale en son pays ${ }^{16}$, souhaitait voir " associer enfin plus intimement l'épigraphie à la paléographie et porter plus spécialement l'effort sur une étude morphologique des inscriptions médiévales ".

Si la définition de l'épigraphie semble devoir être fondée sur le contenu plutôt que sur la forme, les objectifs de l'épigraphie paraissent aussi devoir être commandés en premier lieu par ce contenu, c'est-à-dire par cet élément d'information qu'à un moment donné on a voulu porter à la plus large connaissance, ou par ces éléments intrinsèques qui se révèlent à nous à l'insu peut-être des auteurs de ces inscriptions : culture - ou absence de culture - de ceux qui les ont composées, société à laquelle elles s'adressaient, spiritualité dont elles sont souvent un témoignage direct (culte des saints, sentiment religieux devant la mort), notion de publicité, etc., et de façon générale par la recherche d'une meilleure connaissance du

13. Cette notion est clairement soulignée par I. RoBERT, Epigraphie, dans "I,'histoire et ses méthodes", Paris, I96r, p. 458-459 (a Iincyclopédie de la Pléiade ").

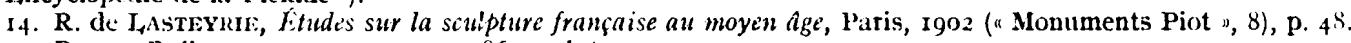

15. Dans "Bull. monum. ", 1929, p. 5-86, 35 h.t.

I6. J. Stinnon, Oî en sont les études d'épigraphie médiéiale en Belgique? dans "Ann. Fédér. histor. et archéol. de Belgique ", $35^{\circ}$ congrès, Courtrai, 1953, p. 697-705. 
monde médiéval. La forme même de l'inscription peut aussi contribuer à cette connaissance, qu'il s'agisse de l'emplacement des inscriptions, de leur style, de leur technique. Ainsi l'influence byzantine se traduit souvent par des inscriptions placées dans le champ et verticalement. Au tympan d'Autun, Gislebert a étroitement associé la distribution des inscriptions à sa composition, témoignage d'une œuvre mûrement élaborée ${ }^{17}$, alors que dans nombre de peintures murales les inscriptions sont placées dans le champ, au hasard de la place disponible, ce qui tendrait au contraire à montrer que le peintre, inscrivant sur le mur sa "leçon " illustrée, n'avait pas toujours le sentiment de réaliser une " œuvre d'art ". Le style même des inscriptions peut témoigner d'influences culturelles : les inscriptions arabo-siciliennes de l'époque des rois normands montrent le maintien de la tradition égyptienne que la domination fâtimide avait imposée à 1'île, ainsi que l'influence syrienne renforcée par les croisades ${ }^{18}$; on constate aussi que les inscriptions du Roussillon sont bien proches de celles de l'Esspagne du Nord. La technique peut également éclairer un moment d'une société, comme l'a montré M. Jean Lafaurie à propos des monnaies des $\mathrm{X}^{\mathrm{e}}$ et $\mathrm{XI}^{\mathrm{e}} \mathrm{S}^{{ }^{19}}$.

Mais qu'il soit indispensable, au préalable, de bien dater les inscriptions (par l'écriture, la langue, la disposition, les formules, etc.), que l'épigraphie puisse être aussi d'un précieux secours pour l'archéologue dans sa datation des monuments et des œuvres d'art objet de son étude, cela est évident. L'épigraphie peut aider directement l'archéologue en lui apportant des noms d'auteur, des dates, en l'aidant à comprendre l'iconographie ${ }^{20}$. Elle peut, par l'examen paléographique, être un utile " auxiliaire "21, comme l'ont montré maints travaux magistraux de Robert de Lasteyrie ou de M. Paul Deschamps. Ainsi donc, si l'établissement d'un tableau chronologique des formes épigraphiques doit constituer un des premiers objectifs de l'épigraphie, que ce soit plutôt une base indispensable qu'une fin en soi. Ceci permettra de serrer de façon plus scientifique les datations, de nuancer les tableaux d'Edmond Le Blant et de M. Paul Deschamps ${ }^{22}$, tableaux - et ce n'est pas là mettre en cause leur immense mérite de pionniers - que les utilisateurs ont nécessairement tendance à employer de façon trop systématique.

Il faudra notamment considérer la paléographie en fonction des régions, des établissements, des supports, des types d'inscription. Le Sud-Est et le Nord-Est ont des formes paléographiques plus classiques, plus régulières, que celles de l'Ouest; l'abbaye de Saint-Benoît-sur-Loire ${ }^{23}$, centre de haute culture, a des inscriptions aux formes beaucoup plus évoluées que celles que l'on trouve généralement aux alentours de l'an IIOO, même si l'on établit des comparaisons avec des inscriptions d'Orléans ; les inscriptions lapidaires, notamment en Provence, sont largement influencées par les inscriptions gallo-romaines nombreuses dans la région ; une épitaphe est souvent moins soignée que l'inscription d'un orfèvre sur un calice ou un crucifix.

17. W. SAVerL,̈̈NDE, Ueber die Komposition des Weltgerichts-Tympanons in Autun, dans "Zeitschr. f. Kunstgesch. "t.XXIX, 1966, p. $261-294$, I 8 ill.

18. J. Sourdet-'Thomine, le style des inscriptions arabo-siciliennes à l'époque des rois normands, dans " Etudes... li.. LEviProvençal n, Paris, 1962, p. 307-315.

19. J. LAFAURIE, Epigraphie monétaire aux $X^{\circ}$ et $X I^{e}$ siècles, dans "Congrès internat. de numismat., Paris, 1953", t. II, Paris, 1957, p. 545-550. H. Fícirtesau a magistralement montré comment l'écriture étail en rapport avec les goúts de chaque époque, la situation de la socićté à un moment donné, le tempérament personnel des scribes, dans Mensch und Schrift im Mittelalter, Vienne, I946.

20. Ainsi les inscriptions ont permis de restituer le sens de la composition des figures peintes dans l'église des Augustins de Toulouse. Cf. P. Mesze et abbé M. Rascol, Les peintures murales de l'église des Augustins de Toulouse, dans "Monum. histor. de la France ", rg6r, p. $167-186$.

2I. L'important est de ne pas cantonner l'épigraphie dans un rôle étroit de science auxiliaire, terme que bannissait déjà Y. RENouARD en 1955 dans son rapport sur les travaux de la section d'études médiévales au Congrès international des sciences historiques : "il est temps de se libérer du titre péjoratif de sciences auxiliaires de l'histoire".

22. E. LF BLANT, Paléographie des inscriptions latines du III' siécle à la fin du VII', dans "Revuc archéologique ", $3^{\mathrm{e}} \mathrm{s}$., $\mathrm{t}$. XXIX, 1896, p. 177-197, 345-355; t. XXX, I897, p. 30-40, I7 I-184; t. XXXI, I 897, p. 172-184. C'est la dernière auvre de Le Blant et elle part de l'ensemble de son cuvre; pourtant $M$. Ch. PERRAT (Paléographie romaine, dans Relazioni del $X$ Congresso intern. di Scienze storiche, I : Metodologia, problemi generali, scienze ausiliari della storia, Florence, 1955, p. 356, n. 3) considere cette étude comme "prématurée et insuffisante ", malgré les services qu'elle rend. Cf. encore P. DESChAMPs, op. cit. ; le compte rendu de M. SchapIRo sur cette dernière étude, dans "Art Bulletin ", t. XII, I930, p. Ior-ro9, à côté d'observations importantes, notamment sur la nécessité d'étudier les inscriptions par "groupes homogènes!,", c'est-à-dire par régions, par monuments, présente le travail d'une façon trop systématique, qui ne tient pas assez compte des nombreuses nuances exprimées par l'auteur dans son exposé.

23. R. Botcher de Molavoox, op. cit. [voir supra, p. 394, 11. 7]. 
Jusqu'ici l'épigraphie médiévale n'a pris un certain essor qu'en Allemagne et en Italie ${ }^{25}$. Llle devrait faire désormais des progrès en France, grâce à l'initiative prise par M. E.-R. I rabande, professeur d'histoire du moyen âge et directeur du Centre d'Études Supérieures de Civilisation Médiévale à Poitiers. En I968 il obtenait du Centre National de la Recherche Scientifique la création d'une équipe de recherche associée pour l'établissement d'un Corpus épigraphique cle la France médiévale, et, du Ministère de l'Éducation nationale, sur proposition du Conseil de la liaculté des Lettres et Sciences humaines de Poitiers, la création à l'Université de Poitiers d'un poste de maitre-assistant, dont le titulaire serait expressément chargé d'un enseignement d'épigraphie médiévale, enseignement qui n'existait jusqu'en I968 ni en France, ni ailleurs, semble-t-il, dans le monde.

Le cours d'épigraphie médiévale a effectivement commencé à fonctionner, au C.É.S.C.M., depuis janvier Ig69.

Le Corpus épigraphique de la France médiévale est placé sous le contrôle d'une commission composée de quatre membres de l'Institut : MM. Paul Deschamps, Jean Hubert, Henri-Irénée Marrou et Charles Samaran. Il doit être réalisé par régions-programme actuelles, à commencer par la région Poitou-Charentes (Charente, Charente-Maritime, Deux-Sèvres, Vienne). La recension bibliographique est achevée pour ces quatre départements, tandis que se poursuit une recension bibliographique générale qui promet d'être surabondante, tant les études d'épigraphie sont ordinairement dispersées et brèves. L'étude sur place, la photographie, la constitution de " dossiers " seront réalisées en I $969 / 70$ pour la Vienne et les Deux-Sèvres, en I970/7 1 pour la Charente et la Charente-Maritime. Chaque dossier doit comprendre les éléments suivants : photographie (ou estampage), aspects extérieurs, texte et traduction ${ }^{28}$, langue, commentaire, bibliographie. L'ensemble sera complété par divers fichiers : noms de personnes et de lieux, matières, citations, et naturellement formes paléographiques.

Le Corpus doit progressivement couvrir toutes les régions de France. Les inscriptions seront classées par départements et par communes, à l'emplacement où elles se trouvent actuellement (cas des musées en particulier). Seront retenues aussi les inscriptions qui ne nous sont plus connues que par des témoignages écrits" ${ }^{27}$. Le terminus a quo doit être le milieu du viII ${ }^{\mathrm{e}} \mathrm{s}$., l'entreprise faisant suite à la refonte du recueil de Le Blant, actuellement en cours sous la direction de M. H.I. Marrou. Le terminus ad quem pourrait être I300. En principe le Corpus recueillera toutes les inscriptions, quel qu'en soit le support matériel, quel qu'en soit l'objet. Toutefois il a été décidé de ne pas retenir systématiquement les légendes des monnaies ni des sceaux, domaine déjà prospecté par les numismates et les sigillographes, ni non plus, pour des raisons matérielles évidentes, les signes lapidaires, dans la mesure du moins où il ne s'agit pas de véritables " signatures "28. Monnaies, sceaux, signes lapidaires n'en seront pas moins utilisés aussi largement que possible à titre de comparaison dans les commentaires, dans le tableau des formes épigraphiques, et sans doute de façon directe dans quelques cas particuliers.

24. F.X. KRAts, Die christlichen Inschriften der Rheinlande..., Fribourg-en-Br., I 890/94, 3 vol. (le L. II étant consacré aux inscriptions chrétiennes du milieu du vسr ${ }^{\mathrm{e}}$ au milieu du $\mathrm{XII}^{\mathrm{e}} \mathrm{s}$.) ; R. CONRARD, Niederrheinische Epigraphik vom achten bis dreizchnten Jahrhundert: cin Beitrag zur Geschichte der monntmentalen Schrift, Francfort, I93I; K. BRaNDI, Grundlegung einer deutschen Inschriftcnkunde, dans "Deutsches Archiv ", t. I, I937, p. II-43. Une publication abondamment illustrée, et qui, surtout, n'est plus limitée à la Rhénanic, est en cours (Die deutschen Inschriften).

25. Relevé établi sous la directlon de A. Silvagn, Monumenta epigraphica christiana saeculo XIII antiquiora quae in Italiae finibus adhuc exstant, iussu Pii XII pontificis maximi, Vatican, t. I, I943, recueil de XLv planches. Voir aussi l'étude de Mme N. ('Bay, The Paleography of Latin Inscriptions: Ninth and Tenth Centuries in Italy, dans a Papers Brit. School Rome ", t. XVI, I948, p. 38-1 7I.

26. "Ia préface de tout commentaire épigraphique devrait être une traduction intégrale "écrivait $\mathrm{S}$. REINACH dans son Traité d'épigraphie grecque, Paris, 1885 , p. Xxx.

27. Les textes d'inscriptions disparues ont été fréquemment écartés par les auteurs de recueils épigraphiques.

28. Parmi les " signes lapidaires " seront écartés les traits, symboles, qui ne représentent vraisemblablement que des naarques de tâcherons. Sera conservé tout ce qui peut être considéré comme une véritable signature, et qui, notamment cromporte dè lettres. I,e partage, il faut bien l'avouer, pourra être malaisé, mais il serait aussi regrettable de laisser de côté entièrement l'apport des signés lapidaires les plus développes, qu'il serait matériellement difficile de recenser la tutalité de ces signes. 
Ainsi les études d'épigraphie médiévale devraient-elles désormais progresser de façon régulière, tandis que se constituera à Poitiers, pour les besoins de la petite équipe d'épigraphistes, un matériel documentaire important qui, conservé au Centre d'Études Supérieures de Civilisation Médiévale, se trouvera naturellement à la disposition des chercheurs qualifiés, en espérant qu'une publication puisse ultérieurement donner à cette source historique, actuellement si difficile à utiliser de par sa dispersion, la plus large des diffusions.

L'épigraphie aborde de nombreux domaines : monuments, peintures murales, vitrail, orfèvrerie, émaillerie, travail du fer, du cuivre, du plomb, du bronze, broderie, tapisserie, bois, cire, ivoire... I es inscriptions intéressent aussi bien les historiens que les archéologues, les philologues, les juristes, les liturgistes, etc. Science de contacts entre historiens, dans le plein sens du mot "interdisciplinaire ", on peut espérer que son apport propre, compris et dans une certaine mesure enrichi par tous les médiévistes, aidera à éclairer notre connaissance de la plus longue période de notre histoire.

*Robert Favreau. 